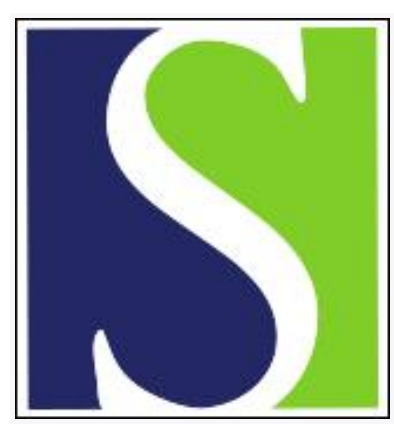

Scand J Work Environ Health 1998;24(4):293-299

https://doi.org/10.5271/sjweh.323

Issue date: Aug 1998

From a unidimensional to a bidimensional concept and measurement of workers' safety behavior

by Marchand A, Simard M, Carpentier-Roy M-C, Ouellet F

Key terms: compliance; concept; factor analysis; manufacturing; reliability; safety initiative; safety rule; worker behavior

This article in PubMed: www.ncbi.nlm.nih.gov/pubmed/9754861

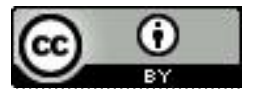




\title{
From a unidimensional to a bidimensional concept and measurement of workers' safety behavior
}

\author{
by Alain Marchand, MSc, ${ }^{1}$ Marcel Simard, PhD, ${ }^{1,2}$ Marie-Claire Carpentier-Roy, PhD, ${ }^{1,3}$ François Ouellet, \\ $M S c^{1}$
}

\author{
Marchand A, Simard M, Carpentier-Roy M-C, Ouellet F. From a unidimensional to a bidimensional concept and \\ measurement of workers' safety behavior. Scand J Work Environ Health 1998;24(4):293-299.
}

\begin{abstract}
Objectives This study examines the concept and measurement of worker's safety behavior. It shows that the traditional concept of safety behavior centered on workers' carefulness or compliance with safety rules is limited and proposes that an additional dimension, namely, workers' safety initiatives, be taken into account.

Methods Confirmatory factor analyses were carried out for a random sample of 828 workers drawn from 9 manufacturing facilities located in the province of Quebec (Canada).

Results A 2-correlated congeneric factor model gave parameters in the expected direction, but the overall model was unable to reach a good fit. Separate construct analyses showed that compliance with safety rules is not a consistent dimension. The safety-initiatives dimension achieved a good fit with a high composite reliability $(\rho=0.85)$.

Conclusions Workers' compliance with safety rules was not structured as a unitary dimension; therefore a selective process of safety-rules compliance by workers is suggested. Each category of safety rules should be considered as 1 single dimension and measured by several specific indicators. Indicators for safety initiatives provide high reliability, and, since this dimension is an important predictor of effectiveness in accident prevention, the items tested provide a better measurement than those previously published.
\end{abstract}

Key terms compliance, concept, factor analysis, manufacturing, measurement, reliability, safety initiative, safety rules, worker behavior.

Understanding how workers behave regarding workplace hazards is an important practical issue in occupational safety since it logically orients actions aimed at developing safety-oriented behavior among the work force. The currently predominant concept of safety behavior is the one stemming from a large number of behavioral safety studies published over the last 2 decades $(1-3)$. In these studies, safety behavior is defined as the degree of compliance with safety rules, hence the implicit assumption that safety behavior is a unidimensional concept.

This is too limited an approach to safety behavior given the results of some other studies. Andriessen (4) has found, with a factor analysis of an 11-item questionnaire administered to a sample of 207 construction workers, that safety behavior has at least 2 dimensions, carefulness and initiatives. Carefulness refers to workers' compliance with safety rules, while initiatives consist of workers' actions to improve the safety of the work environment. Andriessen (4) also showed that these 2 dimensions of worker behavior in occupational safety are positively correlated. More recently, Simard \& Marchand (5, 6) used a similar bidimensional concept of safety behavior in a survey of 1064 work groups nested in 94 Canadian manufacturing firms. They also observed a positive correlation between safety rule compliance and safety initiatives, and, most importantly, their results show that the workers' propensity to take safety initiatives is a major determining factor of workplace effectiveness in accident prevention. Some other studies have also empirically documented the "initiative dimension" in the safety behavior of workers, using labels such as system-oriented strategy (7), "savoir-faire de prudence" $(8,9)$, and auto control $(10,11)$. All these studies provide

1 Health and Prevention Social Research Group (GRASP), University of Montreal, Montreal, Canada.

2 School of Industrial Relations, University of Montreal, Montreal, Canada.

3 Nursing Science Faculty, University of Montreal, Montreal, Canada.

Reprint requests to: Mr Alain Marchand, GRASP, University of Montreal, CP 6128, Succ Centre-ville, Montreal (Quebec), Canada, H3C 3J7. [e-mail: marchand@ere.umontreal.ca] 
empirical evidence supporting the use of at least a bidimensional concept of safety behavior in future research.

The methodological properties of measurement tools used in research can also be improved. Behavioral safety studies have classically used lists of safety rules and trained observers to observe workers randomly in order to measure the degree of compliance before and after treatment (eg, training, goal-setting, feedback). However, reliability tests are not performed on the data collected through observational tools, and we do not know the extent to which the propensity to comply with safety rules, as measured by these tools, form a single dimension of safety behavior. Moreover, check lists of safety rules are rather specific to each workplace, and therefore their external validity should be questioned. Studies using a questionnaire survey approach also have their weaknesses. For example, we do not know the reliability of the measures and factors of Andriessen (4). The reliability test of the compliance and initiative scales of Simard \& Marchand (5) were reported, but they are not very high ( 0.64 and 0.69 , respectively) and the measures were based on supervisors' perceptions of the safety behavior of their employees. The reliability test of the system-oriented strategy scale developed by Brody (7) was better $(0.85)$, but the measure correlated with that of another strategy (problem-focused strategy), and, since Brody (7) did not perform a factor analysis of all the items involved, we do not know if they formed 2 distinct dimensions of safety behavior.

Taking into account these limitations of previous studies and the current interest in a larger approach to safety behavior, particularly in the context of contemporary research on safety culture $(3,12-14)$, we think it is important to improve our analytical conceptualization of workers' safety behavior and the validity of our measurement tools for the benefit of both the research community and occupational safety practitioners. This paper reports results from a confirmatory factor analysis of worker behavior in occupational safety among a random sample of 828 manufacturing workers from 9 manufacturing facilities. The main objective was to try to confirm 2-correlated compliance with the factor structure for safety rules and safety initiatives for measuring worker behavior in occupational safety and provide an estimate of reliability for the resulting scales.

\section{Conceptual definition and hypothesis}

Our bidimensional concept of safety behavior had both theoretical and empirical grounds. Theoretically, it was derived from a sociological approach defining occupational safety as a sociotechnical process of the regulation of hazards produced in the course of economic and work activities $(6,10,15-17)$. This regulation process consists of producing and enforcing social and technical regulations, rules, and standards to control hazardous conditions or behavior in the workplace. Several social actors are involved in this regulation process, for example, government agencies, the business community, labor unions, and scientific and professional communities at the macrosocial level and management, safety practitioners, and the work force at the micro level of each workplace. From this perspective, it is easy to understand why rank-and-file workers' involvement in this regulation process has been primarily conceived traditionally as compliance with safety rules established by other actors. But, at the same time, this regulation approach provides the theoretical rationale enabling us to conceive that workers may also participate in the process of setting up safety rules, which they do when they take safety initiatives. In other words, if one defines worker safety behavior in terms of participation in occupational safety as a sociotechnical regulation process, one is encouraged to use a bidimensional concept of safety behavior that takes the following 2 propensities into account: the propensity to comply with prescribed safety rules and the propensity to take safety initiatives.

Empirical evidence also supports such a bidimensional concept of safety behavior. The compliance-with-safety-rules dimension is a practical issue in most workplaces, given that firms have legal obligations in many countries regarding employees' health and safety at work, which bring them to more or less make and enforce safety rules that should be followed in the performance of a job. The extent to which workers comply with these formal safety rules is the first dimension of the concept of safety behavior, labeled "compliance with safety rules", Previously mentioned studies have also empirically documented workers' behavior regarding safety rules. The most commonly found safety rules in almost all industrial firms are related to the wearing of individual protective devices (boots, gloves, goggles, hearing protector, etc), performing tasks according to safe work methods, use of appropriate tools and equipment, housekeeping of the work station, working at a safe work pace, complying with specific safety procedures (lockout/tagout, work permit, confined space, etc). We have assumed that, when workers comply with 1 or more of these rules, they also tend to comply with the others. Each of the safety rules should thus be linearly related and should form one compliance-with-safety-rules dimension.

The propensity of workers to take safety initiatives is also empirically documented. Various studies, among them the aforementioned, confirm what can be observed in most workplaces. It happens that workers report hazardous situations, make suggestions to improve workplace safety, refuse to perform unusually dangerous tasks, seek information about the risks of particular or unusual 
tasks, and exert pressures of various kinds on management in order to improve safety in the work environment. Again, it is assumed that, when workers take 1 or more of these safety initiatives, they also tend to take other safety initiatives. Each of the safety initiatives should then be linearly related to form 1 safety-initiative dimension.

Finally, from previous studies by Andriessen (4) and Simard \& Marchand (5), it can be hypothesized that safety-rule compliance and safety initiatives are correlated dimensions, in other words, a worker showing more compliance with safety rules will also tend to be more oriented toward safety initiatives and vice-versa. Andriessen (4) reported a moderate correlation of $r=0.34$, while Simard \& Marchand (5) obtained $\mathrm{r}=0.35$.

\section{Material and methods}

\section{Sample}

Data were collected in 1995 in 9 unionized Canadian manufacturing plants of the province of Québec. The facilities were selected in reference to various levels of workplace hazards and accident rates: high level (primary transformation of metals, $\mathrm{N}=3$ ), medium level (metal and mineral products, maintenance services, $\mathrm{N}=5$ ), low level (tobacco products, $\mathrm{N}=1$ ). The 9 plants employed a total of 2143 workers nested in 251 work teams. The plant sizes ranged from 53 to 440 workers with an average of 238.1 and a standard deviation of 127.5.

Within each plant, a random sample of work teams was drawn. When a team was sampled, all workers within it were selected. This procedure yielded representative samples of the work forces of the plants, for a total of 828 workers nested in 126 work teams. All selected work teams were reached, and the overall workers' response rate was $85.4 \%$, similar across plants $\left(\chi^{2}=3.44\right.$, degrees of freedom $(\mathrm{df})=8, \mathrm{P}=0.90)$. The questionnaire took about 45 minutes to be completed. It covered a wide range of health and safety, supervision, work team, task, general organizational context, and sociodemographic variables. The questionnaire was administered under the supervision of a member of the research team.

\section{Measures}

The workers were asked to evaluate the frequency of their behavior for each item on a 5-point Likert-type scale, presumably measuring compliance with safety rules and safety initiatives. The response choices were $1=$ never, $2=$ =very rarely, 3 =sometimes, $4=$ often, $5=$ very often. The set of items referring to compliance with safety rules were (i) wearing of requested individual protective devices (list relevant to each workplace), (ii) use of safe

work methods in the performance of job, (iii) use of appropriate and nondefective tools and equipment, (iv) housekeeping of work station, (v) working according to a safe work pace (no rush), (vi) following safety procedures applicable to specific tasks (list relevant to each workplace). For safety initiatives, the items were (i) reporting a hazardous situation to supervisor, (ii) making a point or a presentation at the safety meeting of the work group, (iii) refusing to do a dangerous job, (iv) reporting a hazardous situation to the safety representative, (v) requesting information about the risk of a particular task from the supervisor, the safety representative, or a coworker, (vi) suggesting a safety improvement or a corrective action to the supervisor, (vii) exerting pressure on the supervisor to follow-up a corrective action.

\section{Statistical analysis}

A confirmatory factor analysis (18-20) was carried out on the 13 manifest variables underlying the compliance with safety rules and safety initiatives of worker behavior in occupational safety. Let the 6 indicators of compliance with safety rules be $\mathrm{x}_{1}, \mathrm{x}_{2}, \ldots, \mathrm{x}_{6}$ and $\mathrm{x}_{7}, \mathrm{x}_{8}, \ldots, \mathrm{x}_{13}$ be the 7 indicators of safety initiatives, and let $\xi_{1}$ and $\xi_{2}$ be the latent variables for compliance with safety rules and safety initiatives, respectively. Furthermore, let $\delta_{1} \ldots \delta_{13}$ be the error variables for $\mathrm{x}_{1} \ldots \mathrm{x}_{13}$. Thereafter, we can write a 2 -factor congeneric-measures model as:

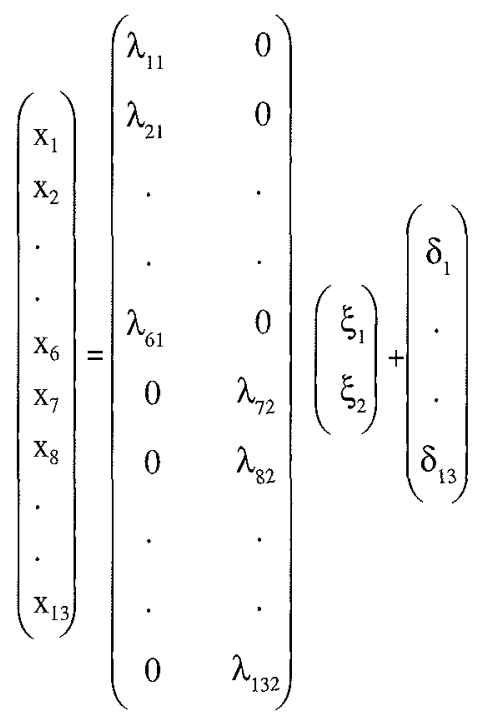

or, compactly, as

$$
\mathrm{x}=\Lambda \xi+\delta
$$

where $\Lambda$ is a $13 \times 2$ factor-loading matrix, $\delta$ are collected in $\Theta$, which is a $13 \times 13$ matrix with error variances in the diagonal, and, since $\xi_{1}$ and $\xi_{2}$ are correlated, the model includes a $2 \times 2 \Phi$ correlation matrix. 
To estimate the 2-factor congeneric-measures model, a polychoric correlations matrix is first computed. This type of correlation, which uses the observed score values to estimate a pair of latent variables that are assumed to have a bivariate normal distribution, is well suited for covariance modeling because regular covariance and Pearson coefficients give biased estimates for ordinal Likert-type scales $(18,19,21-23)$. Second, as the element of the polychoric matrix does not have asymptotic variances and covariances, an asymptotic covariance matrix (W) of the estimated polychorics is computed to obtain correct standard errors and $\chi^{2}(18,21$, $22)$. The polychoric and asymptotic covariance matrices are computed with Prelis 2.1 (24), and parameters of the model are estimated with the weighted least squares (WLS) fitting function embedded in Lisrel 8.1 (25).

The fit of the model to the data is evaluated by relying on $\chi^{2}$, the root-mean-square error of approximation (RMSEA), and the comparative fit index (CFI). $\chi^{2}$ measures the distance between the correlation matrix and the fitted correlation matrix with df (degree of freedom) $=\mathrm{s}-\mathrm{t}$, where $\mathrm{s}=\mathrm{k}(\mathrm{k}+1) / 2, \mathrm{k}=$ number of variables, and $\mathrm{t}=$ the number of estimated parameters (26). The fit of the model is rejected if $\chi^{2}$ has a P-value of $<0.05$. RMSEA (27) is based on the population error of approximation and can be used as a measure of discrepancy per degree of freedom (28). Brown \& Cudeck (28) argued that practical experience led them to consider a RMSEA of $\leq 0.05$ as an indication of close fit for a model. A P-value for testing that RMSEA $\geq 0.05$ could be calculated with the hypothesis of close fit rejected if $\mathrm{P}>0.05$ (28). CFI (29) is bound to lie between $0-1$, and it measures how much better the model fits the data as compared with the independence model. According to Dunn et al (30), CFI needs to have values above 0.90 before the corresponding model can even be considered moderately adequate. At the end, separate construct composite reliability $(\rho)$ will be estimated using equation 4 of Brown (19).

\section{Results}

Table 1 provides the polychoric correlations matrix. Overall, correlations for safety-initiative manifest variables are higher than those for compliance with safety rules.

Table 2 presents the results from the WLS estimation of the 2-correlated congeneric-factors model. All $\lambda$ estimates were statistically significant $(\mathrm{P} \leq 0.01)$ and were in the expected direction, as well as the correlation between the 2 factors. However, the model was unable to reach a good fit, as shown by the fit indices. Error variances were, on the average, greater for compliance with the indicators of safety rules.

The lack of fit of the model may, to some extent, have been caused by the low correlations observed for compliance with safety rules and by the large error variance for most of its indicators. As suggested by Bollen (18), we have run a separate analysis for each factor to investigate the potential source of lack of fit for the model. The results of these analyses are presented in table 3 .

The measurement model for compliance with safety rules does not fit the data, as shown by the fit indices. The indicators appear to be weakly explained by the latent variable, as revealed by low $\lambda$ estimates and consequently large error variances. This result is not surprising considering the low correlations observed for these indicators. Computing composite reliability for this construct gives only $\rho=0.57$.

For the safety initiative dimension, the results showed that the measurement model fit well when RMSEA and

Table 1. Polychoric correlations matrix $(N=828) .^{a}\left(x_{1}=\right.$ wearing individual protective devices, $x_{2}=$ using safe work methods, $x_{3}=u s i n g$ appropriate and nondefective tools and equipment, $x_{4}=$ housekeeping of the work station, $x_{5}=$ safe work pace, $x_{6}=$ following safety work procedures, $x_{7}=$ reporting a hazardous situation to supervisor, $x_{8}=$ making a point or a presentation at the safety meeting, $x_{9}=$ refusing to do a dangerous job, $x_{10}=$ reporting a hazardous situation to the safety representative, $x_{11}=$ requesting information about the risk of a particular task from the supervisor, the safety representative or a co-worker, $x_{12}=$ suggesting a safety improvement or a corrective action

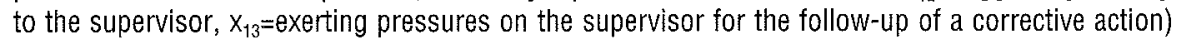

\begin{tabular}{|c|c|c|c|c|c|c|c|c|c|c|c|c|c|}
\hline$x_{1}$ & 1.00 & & & & & & & & & & & & \\
\hline$x_{2}$ & 0.06 & 1.00 & & & & & & & & & & & \\
\hline$x_{3}^{2}$ & 0.18 & 0.08 & 1.00 & & & & & & & & & & \\
\hline$x_{4}^{0}$ & 0.07 & 0.33 & 0.09 & 1.00 & & & & & & & & & \\
\hline$x_{5}$ & 0.16 & 0.20 & 0.21 & 0.20 & 1.00 & & & & & & & & \\
\hline$x_{6}$ & 0.25 & 0.11 & 0.17 & 0.20 & 0.14 & 1.00 & & & & & & & \\
\hline$x_{7}$ & 0.03 & 0.22 & 0.00 & 0.23 & 0.11 & 0.12 & 1.00 & & & & & & \\
\hline$x_{8}$ & 0.01 & 0.25 & -0.06 & 0.14 & 0.08 & 0.13 & 0.42 & 1.00 & & & & & \\
\hline$x_{9}$ & 0.03 & 0.21 & -0.12 & 0.13 & 0.07 & 0.07 & 0.33 & 0.28 & 1.00 & & & & \\
\hline$x_{10}$ & 0.09 & 0.18 & -0.05 & 0.16 & 0.00 & 0.15 & 0.46 & 0.33 & 0.46 & 1.00 & & & \\
\hline$x_{11}$ & 0.03 & 0.16 & -0.07 & 0.14 & 0.06 & 0.17 & 0.31 & 0.32 & 0.40 & 0.44 & 1.00 & & \\
\hline$x_{12}$ & -0.01 & 0.27 & -0.07 & 0.38 & 0.08 & 0.09 & 0.58 & 0.49 & 0.40 & 0.46 & 0.45 & 1.00 & \\
\hline$x_{13}$ & 0.04 & 0.22 & -0.10 & 0.22 & 0.13 & 0.15 & 0.48 & 0.35 & 0.44 & 0.40 & 0.37 & 0.56 & 1.00 \\
\hline
\end{tabular}

a Correlations $\geq 0.09$ are $P \leq .0 .05$, Correlations $\geq 0.12$ are $P \leq 0.01$. 
CFI were considered. $\chi^{2}$ was however significant, but the test was rigid since it implied an exact reproduction of, in our case, the correlation matrix, it ignored the statistical power of the test, and failure of the variable distributional assumptions could lead to the rejection of correct models or the failure to reject incorrect models (31). The model was thus considered to have a good fit for the data with a high construct composite reliability of $\rho=0.85$.

The last analysis was exploratory in nature and started with the hypothesis that workers have a specific behavior for each safety rule highlighted by indicators of the hypothesized compliance-with-safety-rules dimension. In other words, when workers follow a specific rule, it does not mean that they will follow the other rules. Thus, behavior regarding each rule can be viewed as being a single dimension, although some rules may be correlated to some extent. We then performed an analysis with 7 correlated factors where the first 6 factors represented each safety rule previously defined with $\lambda_{11}$ thru $\lambda_{66}$ fixed at 1 and $\delta_{1}$ to $\delta_{6}$ fixed at 0 . The last factor was the safety initiative factor. Estimating this model gave a better fit for the data with $\chi^{2}=117.86, \mathrm{df}=56, \mathrm{P}=0.00$, RMSEA $=0.037 \mathrm{P}(\mathrm{RMSEA}<0.05)=0.99$, and $\mathrm{CFI}=0.94$. From the 2-correlated factors model, the 7-correlated factors model represented an improvement of $\chi^{2}=112.73$, $\mathrm{df}=8, \mathrm{P}=0.00$ in the fit, which was highly significant. This exploratory analysis thus somewhat supported a model of worker behavior in occupational safety with a safetyinitiatives behavior factor and specific behavior regarding safety rules.

\section{Discussion}

The first objective of this paper was to try to confirm a 2-correlated factors model capturing worker safety behavior in terms of compliance with safety rules and safety initiatives. Our analysis of the 13 indicators revealed that a 2-correlated factor model was unable to reach a good fit for the data. The lack of fit of the model was investigated by performing a separate analysis for each construct, and we found that the measurement model did not hold for compliance with safety rules while the measurement model for safety initiatives fit well.

According to our results, compliance with safety rules does not appear to be structured as a unitary dimension. Workers seem to have a specific behavior regarding each of the safety rules listed. This finding can be interpreted as revealing a pattern of behavior whereby workers are selective regarding the safety rules they comply with. This selection process is probably based upon workers' own evaluation of the adequacy and relevance of the rule, which may be influenced by various factors. One is the workers' own cost-benefit analysis of the safety rule,
Table 2. Weighted-least-squares results for the 2-correlated congeneric factor model $(\mathrm{N}=828)$. $(\mathrm{df}=$ degrees of freedom; RMSEA = root-mean-square error of approximation; $\mathrm{CFI}=$ comparative fit index; see table 1 for the $x_{1}$ to $x_{13}$ descriptives)

\begin{tabular}{lcccc}
\hline Indicators & $\begin{array}{c}\text { Compliance } \\
\text { with safety } \\
\text { rules }\left(\xi_{1}\right) \\
\lambda\end{array}$ & $\begin{array}{c}\text { Safety } \\
\text { initiatives }\left(\xi_{2}\right)\end{array}$ & $Z(\lambda / \mathrm{s} . \mathrm{e})$ & $\delta$ \\
\hline$x_{1}$ & 0.31 & & & \\
$x_{2}$ & 0.58 & & 6.90 & 0.90 \\
$x_{3}$ & 0.23 & & 14.22 & 0.66 \\
$x_{4}$ & 0.63 & & 5.66 & 0.95 \\
$x_{5}$ & 0.43 & & 16.72 & 0.60 \\
$x_{6}$ & 0.44 & 0.71 & 10.11 & 0.82 \\
$x_{7}$ & & 0.56 & 18.02 & 0.80 \\
$x_{8}$ & & 0.57 & 17.53 & 0.50 \\
$x_{9}$ & & 0.63 & 20.63 & 0.69 \\
$x_{10}$ & & 0.58 & 20.53 & 0.67 \\
$x_{11}$ & & 0.81 & 38.11 & 0.61 \\
$x_{12}$ & & 0.70 & 26.78 & 0.34 \\
$x_{13}$ & & & 14.84 & 0.51 \\
$\Phi\left(\xi_{1}, \xi_{2}\right)^{a}$ & & & & \\
\hline
\end{tabular}

a Goodness of fit: $\chi^{2}=230.59, \mathrm{df}=64, P=0.00$; RMSEA $=0.056$; $P(R M S E A<0.05)=0.10 ; C F I=0.85$

Table 3. Weighted-least-squares results for the separate construct congeneric factor model $(\mathrm{N}=828)$. ( $\mathrm{d} f=$ degrees of freedom; RMSEA = root-mean-square error of approximation; $\mathrm{CFI}=$ comparative fit index; see table 1 for the $x_{1}$ to $x_{13}$ descriptives)

\begin{tabular}{lcccc}
\hline Indicators & $\begin{array}{c}\text { Compliance } \\
\text { with safety } \\
\text { rules }\left(\xi_{1}\right)^{2}\end{array}$ & $\begin{array}{c}\text { Safety } \\
\text { initiatives }\left(\xi_{2}\right)^{b}\end{array}$ & $Z(\lambda /$ s.e $)$ & $\delta$ \\
& $\lambda$ & & & \\
\hline$x_{1}$ & 0.36 & & 6.56 & 0.87 \\
$x_{2}$ & 0.45 & & 7.99 & 0.80 \\
$x_{3}$ & 0.33 & & 6.87 & 0.89 \\
$x_{4}$ & 0.52 & & 10.19 & 0.73 \\
$x_{5}$ & 0.46 & & 9.22 & 0.79 \\
$x_{6}$ & 0.42 & & 8.09 & 0.82 \\
$x_{7}$ & & 0.67 & 18.91 & 0.55 \\
$x_{8}$ & & 0.57 & 17.60 & 0.68 \\
$x_{9}$ & & 0.60 & 17.46 & 0.65 \\
$x_{10}$ & & 0.68 & 20.70 & 0.54 \\
$x_{11}$ & & 0.60 & 19.34 & 0.64 \\
$x_{12}$ & & 0.80 & 35.24 & 0.36 \\
$x_{13}$ & & 0.71 & 25.56 & 0.50
\end{tabular}

a Goodness of fit: $\chi^{2}=43.71 \mathrm{df}=9 \mathrm{P}=0.00 ; \mathrm{RMSEA}=0.068$;

$\mathrm{P}(\mathrm{RMSEA}<0.05)=0.06 ; \mathrm{CFI}=0.75$.

b Goodness of fit: $\chi^{2}=47.73 \mathrm{df}=14 \mathrm{P}=0.00$; RMSEA $=0.054$; $\mathrm{P}(\mathrm{RMSEA} 00.05)=0.32 ; \mathrm{CFl}=0.96$

whereby they estimate potential disadvantages of complying with the rule (eg, additional efforts or extended time to do the job, self-image, etc) compared with potential advantages (eg, avoidance of a highly probable or severe injury, reduction of job stress, etc) (32). Another factor is social pressure either from the supervisor or the work group. For example, safe work methods are generally managerial standards defining how workers should perform tasks in order to prevent injury. If management properly enforces these standards (eg, training, supervision, reinforcement), pressure is exerted on the workers to use the standards in the performance of their 
tasks. Strong and cohesive work groups also set and enforce informal work methods that may differ from managerially defined work standards $(8,10,33,34)$. Naturally, the combination of the factors may differ from one safety rule to another, which may explain why a worker may, for example, wear his personal protective devices but rarely use work standards in the performance of tasks, in other words, not have a consistent behavior in terms of compliance with safety rules.

Thus the bad fit of the model and the poor reliability obtained for a global measure of compliance with safety rules $(\rho=0.57)$ brings us to propose a reconceptualization of this dimension. Based on our exploratory results for a 7-correlated factors model for worker-safety behavior, each category of safety rules (eg, personal protective devices, safe work methods, etc) should be considered as a single dimension, measured by several specific indicators rather than only by one, as we did, and moderate correlations between these dimensions, as we observed, would only indicate that some people do comply with more than 1 safety rule, however, without there being a consistent tendency in samples. At this point, this proposal to conceptualize compliance behavior by categorizing safety rules seems to be the most appropriate approach with which to investigate the specific pattern of factors related to each category of safety-rule compliance.

On the contrary, we found a good measurement model for behavior with respect to safety initiatives, with a high composite reliability $(\rho=0.85)$. Of course, this value would have been lower if we had used the regular covariance matrix or the Pearson correlation matrix and had computed Cronbach alpha $(\alpha)$. For our data, the regular Cronbach alpha analysis of the Pearson correlation matrix for the items gave $\alpha=0.81$, which is also good. The safety initiative items tested are thus useful for constructing a scale, and they perform better than the ones previously used by Simard \& Marchand (5), which had $\alpha=0.69$ and were based on supervisors' perceptions of the behavior of their employees. Thus, given that workers' propensity to take safety initiatives is an important variable predicting organizational performance in occupational safety $(5,6)$, a better safety initiative scale should provide better and more reliable results in future research trying either to estimate the impact of that variable on accident prevention or to identify factors explaining this component of worker safety behavior. The next step in the development of a valid measure of safety initiative behavior should be to perform additional analyses to evaluate the invariance of the single safety-initiative congeneric factor model across samples of workers from different occupations, industrial sectors, and countries.

Finally, some limitations of this study must be pointed out. First, the analysis was limited to a small subset of the overall worker population in manufacturing, and it is thus representative only of the 9 firms included in the study. Second, the analysis did not fully recognize the hierarchical nature of the sample, where workers are nested within their work teams. Although multilevel confirmatory factor analysis, based on the random effect approach, is now available for dealing with this kind of problem $(35,36)$, the procedure can only be applied at this point to metric variables. It is not possible to incorporate ordinal variables and the appropriate $W$ matrix since a $\mathrm{W}$ matrix would have to be developed for the between-group components of the model. Another way of handling the hierarchical structure of the data would be to use the pooled within-group covariance matrix, but, again, it would be necessary to develop the appropriate W matrix.

\section{References}

1. McAfee RB, Win AR. The use of incentatives/feedback to enhance work place safety: a critique of literature. J Saf Res 1989;20:7-19.

2. Peters R. Strategies for encouraging self-protective behavior. J Saf Res 1991;22:53-70.

3. Ray PS, Purswell JL, Bowen D. Behavioral safety program: Creating a new corporate culture. Int J Ind Ergon 1993;12:193-198.

4. Andriessen JHTH. Safe behaviour and safety motivation. $J$ Occup Accid 1978;1:368--76.

5. Simard M, Marchand A. The behaviour of first-line supervisors and effectiveness in occupational safety. Saf Sci 1994;19:169-84.

6. Simard M, Marchand A. Participation des travailleurs à la sécurité et taux d'accident du travail. Psychol Trav Org 1996;2:34-44.

7. Brody JG, Responses to collective risk: appraisal and coping among workers exposed to occupational health hazards. Am J Community Psychol 1988;16:645-63.

8. Carpentier-Roy MC. Corps et âme: psychopathologie du travail infirmier. Montréal: Edition Liber, 1995.

9. Dejours C. Intelligence ouvrière et organisation du travail. Paris: Ed. L'Harmattan, 1992

10. Dwyer T. Life and death at work: industrial accidents as a case of socially produced error. New York (NY): Plenum Press, 1992.

11. Eakin, J. Leaving it up to the workers: sociological perspective on the management of health and safety in small workplaces. Int J Health Serv 1992;22:689-704.

12. Peterson D. Establishing good "safety culture" helps mitigate workplace dangers. Occup Health Saf 1993;62:20-4.

13. Pidgeon NE. Safety culture and risk management in organizations. J Cross-Cultural Psychol 1991;22:129-40.

14. Saari J. Successful implementation of Occupational health and safety programs in manufacturing for the 1990 s. Int $\mathbf{J}$ Hum Factors Manuf 1992;2:55-66.

15. Dawson S, Willman P, Bamford M, Clinton A. Safety at work: the limits of self-regulation. Cambridge: Cambridge University Press, 1988.

16. Kelman S. Regulating America, regulating Sweden: a comparative analysis of occupational safety and health policy. 
Cambridge: MIT Press, 1981

17. Perrow C. Normal accidents. New York (NY): Basic Books, 1984.

18. Bollen KA. Structural equation with latent variables. New York (NY): Wiley, 1989.

19. Brown RL. Using covariance modeling for estimating reliability on scales with ordered polytomous variables. Educ Psychol Meas 1989;49:385-98.

20. Joreskog KG, Sorbom D. LISREL 7 user's reference guide. Chicago (IL): Scientific Software Inc, 1989.

21. Joreskog KG. On the estimation of polychoric correlations and their asymptotic covariance matrix. Psychometrika 1994;59:381-9.

22. Joreskog KG, Sorbom D. PRELIS. A preprocessor for Lisrel. Chicago (IL): Scientific Software Inc, 1988.

23. Ridgeon EE, Ferguson CE. The performance of the polychoric correlation coefficient and selected fiting functions in confirmatory factor analysis with ordinal data. J Mark Res 1991;28:491-7.

24. Joreskog KG, Sorbom D. PRELIS 2 user's reference guide Chicago (IL): Scientific Software International Inc, 1993.

25. Joreskog KG, Sorbom D. LISREL, 8 user's reference guide Chicago (IL): Scientific Software International Inc, 1993.

26. Joreskog KG, Sorbom D. LISREL 8: structural equation modeling with the simplis command language. Chicago (IL): Scientific Software International Inc, 1993.
27. Steiger JH. Structural model evaluation and modification: an interval estimation approach. Multivariate Behav Res 1990;25:173-80.

28. Brown MW, Cudeck R. Alternative ways of assessing model fits. In: Bollen KA, Long JD, editors. Testing structural equation models. Newbury Park (NJ): Sage Publications Inc, 1993:136-62.

29. Bentler PM. Comparative fit indexes in structural models. Psychol Bull 1990;107:238-46.

30. Dunn G, Everitt B, Pickles A. Modeling covariance and latent variables using EQS. London: Chapman \& Hall, 1993.

31. Bollen KA, Long JD. Introduction. In: Bollen KA, Long JD, editors. Testing structural equation models. Newbury Park (NJ): Sage Publications Inc, 1993:1-9.

32. Hale AR, Glendon AI. Individual behavior in control of danger. Amsterdam: Elsevier, 1987.

33. Cru D, Dejours C. Les savoirs-faire de prudence dans les métiers du bâtiment. Cah Méd-Soc 1983;3:239—47.

34. Hass J. Real feeling: a study of high stell ironworkers' reactions to fear and danger. Sociol Work Occup 1977;4:147 —70.

35. Muthen BO. Latent variable modeling in heterogeneous population. Psychometrika 1989;54:557-85.

36. Muthen BO. Multilevel covariance structure analysis. Sociol Methods Res 1994;22:376-98.

Received for publication: 4 November 1997 